\title{
Subsistema de Saúde Suplementar: Ensino em Cursos de Graduação na Área da Saúde
}

\author{
Carla Aparecida Arena Ventura*, Rachel Torres Salvatori, Bruna Sordi Carrara, Juliana \\ Cristina dos Santos, Liane Cristina Toyonaga da Silva
}

Escola de Enfermagem de Ribeirão Preto da Universidade de São Paulo

* Autora para correspondência: caaventu@eerp.com.br

\section{RESUMO}

O subsistema de saúde suplementar brasileiro é pouco explorado nos cursos de graduação na área da saúde. $\mathrm{O}$ objetivo deste estudo foi identificar se tal temática está presente nos currículos dos cursos de graduação na área da saúde da USP/Ribeirão Preto. Para a coleta de dados, utilizou-se a análise documental, realizada por meio do exame dos currículos dos nove cursos de graduação na área da saúde da USP/Ribeirão Preto, por meio do sistema Jupiterweb. Foram coletadas 61 ementas dentre as 1218 existentes nas bases do sistema. As ementas de todas as disciplinas dos referidos cursos foram identificadas com as palavras-chave relacionadas à temática. Verificou-se que a grade curricular da maioria dos cursos não oferece conteúdo teórico aos alunos em relação ao subsistema de saúde suplementar. A revisão das ementas das disciplinas mostrou que o assunto não está sendo contemplado. Entende-se que existe uma necessidade de se repensar o currículo, com a introdução dessa temática, visto que é fundamental que o aluno de um curso da saúde, futuro profissional nessa área, conheça as características do sistema para que possa operar corretamente o direito à saúde.

Palavras-Chave: Subsistema de Saúde Suplementar; Grade Curricular; Ensino de Graduação.

\begin{abstract}
The brazilian supplementary health subsystem is not well explored in undergraduate courses in the health area. The objective of this study was to identify if the supplementary health subsystem theme is present in the curriculum of undergraduate courses in the health area of USP/Ribeirão Preto. For the data collection, the documentary analysis was carried out by means of the analysis of the curriculum of the nine undergraduate courses in the health area of USP/Ribeirão Preto, through the Jupiterweb system. A total of 61 menus were collected from among the 1,218 existing in the database. The menus of all disciplines of these courses were identified with the keywords related to the subject. The review of the disciplines' menus showed that the teaching of the supplementary health subsystem is not being considered. It is understood that there is a need to rethink the curriculum, with the introduction of this subject, since it is fundamental that the student of a course of the health area, future professional in this area, knows the characteristics of the system so that he/she can correctly operate the right to health.
\end{abstract}

Keywords: Supplementary Health Subsystem; Curricular Grade; Undergraduate Teaching.

\section{Introdução}

Este estudo faz parte da pesquisa intitulada

O Ensino do Subsistema de Saúde Suplementar nos Cursos de Graduação na Área da Saúde do Campus da USP de Ribeirão Preto, desenvolvida no âmbito do programa Ensinar com Pesquisa por alunas do curso de bacharelado em Enfermagem da Escola de Enfermagem de Ribeirão Preto da Universidade de São Paulo (EERP/USP).
O programa Ensinar com Pesquisa integra a política de valorização do ensino de graduação por meio de um conjunto de ações destinadas ao apoio à formação estudantil na Universidade de São Paulo. Possui como foco o ensino de graduação desta instituição e seus múltiplos aspectos (ambiente da sala de aula, curso, questões curriculares, modalidades de ensinar e aprender, estágio curricular ou profissional, tecnologias de ensino, resultados de 
ensino, inserção profissional de egressos, evasão, entre outros). Nesse contexto, o presente estudo embasou-se na análise da grade curricular de cursos da área da saúde do campus da USP de Ribeirão Preto, a fim de identificar a existência do ensino do Subsistema de Saúde Suplementar.

O subsistema de saúde suplementar brasileiro, por meio de planos privados de saúde, foi iniciado no país na década de 1960. Contudo, esse subsistema ainda é pouco explorado nos cursos de graduação na área da saúde. O grande pilar é o ensino sobre o Sistema Único de Saúde e, dessa forma, os alunos da graduação se formam com muitas lacunas na compreensão daquele subsistema e, muitas vezes, com entendimentos equivocados e distorcidos sobre tal realidade.

No Brasil, uma característica do sistema de saúde, anterior ao marco constitucional de 1988, era a livre atuação entre iniciativas públicas e privadas, no que tange ao oferecimento, ao financiamento e à operação dos serviços de saúde. Com a promulgação da Constituição de 1988, a saúde passa a ser, constitucionalmente, "livre à iniciativa privada" (BRASIL, 1990), sendo estabelecida, ao Poder Público, a competência para sua regulamentação, fiscalização e controle, nos âmbitos público e privado.

Na década de 1960, devido ao aumento crescente do setor privado no financiamento e na oferta dos serviços de saúde, por meio de empresas que intermediavam o pagamento dos serviços dispensados às pessoas que os contratavam, estimuladas pelo crescimento do parque automobilístico, o Estado, que acabou por se afastar, em parte, da prestação da assistência à saúde, passou a assumir mais um papel, na regulação desse tipo de assistência oferecida por meio de empresas privadas.

Assim, nasceram os planos de saúde no Brasil e o subsistema de saúde suplementar. Sato (2007) descreve que a expansão do setor suplementar de assistência à saúde (setor caracterizado pela operação de planos de saúde) foi acompanhada pelo aumento de distúrbios nas relações entre usuários e operadoras de planos de saúde, vinculados, principalmente, às negativas de coberturas assistenciais (materiais, medicamentos, limitação quantitativa de procedimentos, limitação do tempo de internação, inclusive em Unidade de Terapia Intensiva - UTI), ao aumento nos preços das mensalidades dos planos de saúde e à interrupção de atendimento. Como resultado, entidades médicas, órgãos de defesa do consumidor e organizações não governamentais de usuários e consumidores aliaram-se, e, juntamente, com o Conselho Nacional de Saúde, Ministério Público e operadoras de planos de saúde, passaram a exercer uma pressão social para a existência de uma regulamentação específica para o setor (CARVALHO \& GEGíllo, 2007).

Após quarenta anos de livre atuação das operadoras de planos de saúde sem qualquer regulamentação estatal, em 1998, foi promulgada a Lei 9.656, que dispõe sobre os planos e seguros privados de assistência à saúde (BRASIL, 1998), trazendo uma série de obrigações para as empresas que atuavam como operadoras de planos de saúde. Pouco mais de um ano depois de vigência da Lei 9.656/98, foi publicada a Lei 9.961/2000, que cria a Agência Nacional de Saúde Suplementar - ANS, estabelecendo suas competências como agente regulador, normatizador e fiscalizador da operação de planos de saúde (BRASIL, 2000).

$\mathrm{O}$ que se percebia nesse momento no subsistema de saúde suplementar era uma grande variedade de serviços, com padrões de qualidade distintos. A fragmentação da atenção compromete a eficiência do serviço de saúde e atinge as redes de cuidados básicos, especializados e hospitalares, que atendem os clientes dos planos de saúde (PIETROBON et al., 2008).

O subsistema de saúde suplementar é, hoje, dessa maneira, responsável pelo atendimento de quase 30\% da população brasileira e está marcado por relações assimétricas de informação e de poder entre seus atores. De um lado, as operadoras de planos de saúde querem arrecadar o máximo possível e oferecer o mínimo legal; de outro, os prestadores querem a utilização das tecnologias mais modernas e sofisticadas e um alto faturamento; ainda, de um terceiro lado estão os beneficiários dos planos, querendo pagar o mínimo e ter o 
máximo em termos de assistência à sua saúde (PIETROBON et al., 2008).

Diante dessa realidade, a formação em saúde no Brasil e o subsistema de saúde suplementar foram objeto de preocupação e investigação em 2008, em um projeto de pesquisa desencadeado no contexto da cooperação internacional entre a Organização Pan-Americana de Saúde - OPAS - e a ANS, com o objetivo de ampliar o conhecimento e a análise do setor de saúde suplementar. A pesquisa revelou que os estudantes e docentes dos cursos de graduação estudados idealizam uma atuação liberal-privatista, aquela de atendimento individualizado, em consultório particular, de livre arbítrio de profissionais e usuários, combinada com o exercício da profissão no segmento público-estatal (OPAS/ANS, 2009).

O subsistema de saúde suplementar exerce marcante presença no sistema de saúde brasileiro, respondendo por mais de um quarto da cobertura populacional do país. Está em contínua expansão, o que foi verificado por meio do aumento do faturamento das operadoras de planos de saúde e incremento nos planos coletivos, e é um importante demandante e incorporador de tecnologias em saúde. Além disso, é marcado por relações comerciais de poder entre seus atores (operadoras, profissionais da área da saúde, hospitais, clínicas, laboratórios, beneficiários) e é interventor do processo saúde-doença dos beneficiários dos planos privados. $\mathrm{O}$ subsistema também causa interferência na economia do país, fundamentalmente no tocante à renúncia fiscal (já que parte do gasto com planos de saúde pode ser abatida do imposto de renda) e, principalmente, por ser parte do sistema de saúde brasileiro. Sendo assim, torna-se importante e oportuno, nesse contexto, o desenvolvimento de ações que busquem aproximar os alunos dos cursos de graduação na área da saúde da construção do conhecimento no campo do subsistema de saúde suplementar.

\section{Método}

Neste estudo, foi utilizado o método quantitativo, buscando-se atingir uma amostra relevante referente aos cursos de graduação na área de saúde. Além disso, o método quantitativo se preocupa em descrever, diferenciar ou examinar associações, em vez de procurar relações diretas de causa e efeito entre variáveis, grupos ou situações, sendo uma abordagem adequada para pesquisas descritivas (OLIVEIRA, 1999). Portanto, trata-se de estudo quantitativo descritivo-exploratório. Pesquisas descritivas buscam descrever as características de determinada população ou fenômeno, assim como estabelecer relações entre variáveis (GIL, 1994).

Os dados foram coletados por meio de análise documental a qual foi realizada tomando como base os currículos dos cursos de graduação na área da saúde (Medicina, Enfermagem, Odontologia, Farmácia, Nutrição, Fisioterapia, Fonoaudiologia, Terapia Ocupacional e Psicologia) da USP/Ribeirão Preto, por meio do sistema Jupiterweb, sistema on-line com informações referentes aos cursos de graduação da universidade. Foram coletadas 61 ementas dentre as 1.218 existentes nas bases do referido sistema. As ementas de todas as disciplinas dos referidos cursos foram identificadas com as palavras-chave: "seguros de saúde" "seguros médicos privados", "planos de saúde", "saúde suplementar", "sistema ou subsistema de saúde suplementar", "sistemas de saúde" e "serviços de saúde". Os dados foram coletados por meio de roteiro estruturado e analisados por meio da estatística descritiva, através do programa estatístico Epi Info, v.6.04, e pela análise de conteúdo de Bardin (1977).

\section{Resultados e Discussão}

Durante a coleta de dados realizada na base documental, ou seja, nas ementas disponíveis no sistema Jupiterweb, verificou-se a necessidade de ampliar o repertório de palavras-chave inicialmente propostas. No primeiro momento, as palavras procuradas foram "seguros de saúde", "seguros médicos privados", "planos de saúde", "saúde suplementar", "sistema ou subsistema de saúde suplementar". Como não foram encontradas disciplinas cujas ementas tivessem essas palavras, 
posteriormente se adicionaram as palavras-chave "sistemas de saúde" e "serviços de saúde", também relacionadas com a temática deste estudo.

Das ementas pesquisadas, 58 apresentaram alguma palavra-chave das utilizadas para a busca. Dessas, 3,44\% mencionavam "sistemas de saúde", 3,44\% estavam relacionadas a "sistemas de saúde" e "serviços de saúde", simultaneamente, e 93,11\% a "serviços de saúde", como indica o Fluxograma 1.

Esses resultados representam um forte indicativo da pouca ênfase que vem sendo dada ao ensino do tema subsistema de saúde suplementar nesses cursos, uma vez que, se foi contemplado o referido conteúdo, não foi utilizada sequer sua denominação correta.

Segundo Albuquerque (2009), a formação de ensino superior dos profissionais da saúde foi construída com base na fragmentação de conteúdos disciplinares. Fundamentado em Mosé (2008), o autor supracitado compara a formação superior dos profissionais da saúde com o modelo industrial, afirmando que o ensino muitas vezes é considerado uma linha de montagem, em que as diversas disciplinas, em alguns casos, não possuem conexão entre si e podem fazer parte de um mundo distante do estudante. Esse olhar fragmentado que se inicia durante a formação acaba acompanhando o profissional. Nesse contexto, a fragmentação do conhecimento pode impulsionar a formação de profissionais especialistas, ou seja, com uma prática voltada prioritariamente para sua disciplina e que pode ter dificuldade para lidar com problemas mais coletivos, com uma visão mais holística, ao passo que uma perspectiva interdisciplinar, que articula mais profundamente a teoria e a prática, propõe-se a formar um profissional mais generalista e reflexivo, que tem mais facilidade para focar o coletivo e não apenas pensar e planejar no âmbito individual.

Entende-se a interdisciplinaridade como uma atitude pautada pelo rompimento com a postura positivista de fragmentação, objetivando uma compreensão mais ampla da realidade. Com essa atitude, facilita-se uma interação efetiva entre as disciplinas a partir do momento em que se reconhecem os limites das distintas áreas do saber (COUTO, 1997). De acordo com Morin (2008), a educação não deveria formar especialistas fechados em seus saberes, segmentados e separados do todo, mas é importante que prime pela responsabilidade e solidariedade com o todo,

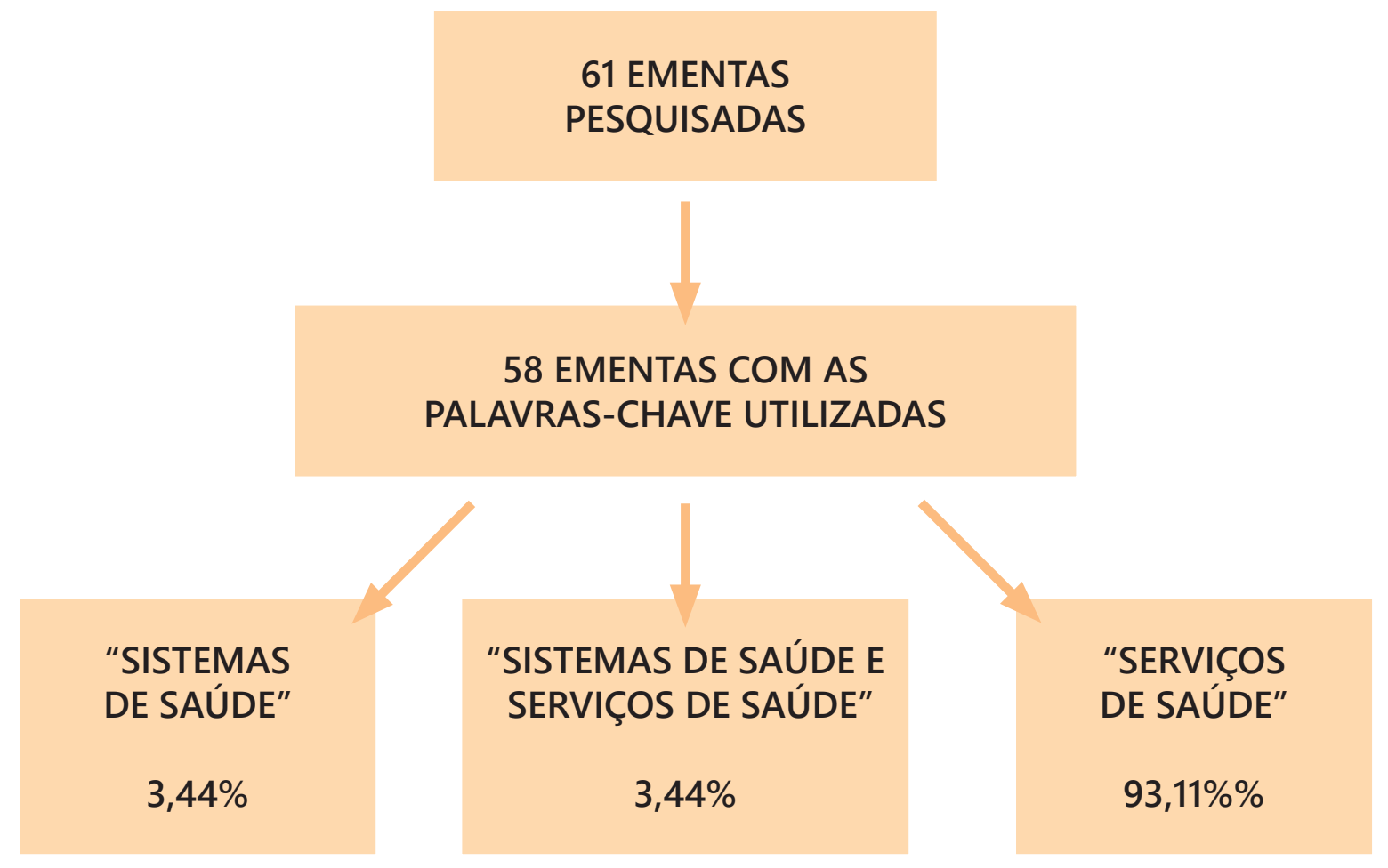

Fluxograma 1 - Resultado das ementas analisadas por palavras-chave. 
além de ser capaz de formar pessoas aptas a trocarem, colaborarem e, especialmente, fazerem ciência com consciência.

Sendo assim, há uma tendência à supervalorização de disciplinas voltadas à área biológica, como anatomia e fisiologia, as quais, na maioria das vezes, ganham um espaço maior na grade curricular, em detrimento de disciplinas com enfoque mais político e social, que buscam entender os princípios que fundamentaram a criação e a implementação do sistema de saúde brasileiro, incluindo o subsistema de saúde suplementar (ALBUQUERQUE et al., 2009).

A formação universitária historicamente vem focando a separação dos objetos de seus contextos. Em muitos casos, conteúdos se dividem em disciplinas que não se integram e que, ao invés de facilitar, acabam por dificultar a compreensão da complexidade da realidade (MORIN, 2008).

Como o grande pilar dos cursos de saúde é o Sistema Único de Saúde, os alunos de graduação se formam com muitas lacunas na compreensão do subsistema suplementar. Além disso, com bastante frequência, o primeiro empregador dos recém-egressos dos cursos de graduação na área da saúde é o prestador privado de saúde, dependente financeiramente, em sua grande maioria, dos planos de saúde (SALVATORI, 2013).

A investigação da formação de alunos de cursos da área da saúde sobre seus conhecimentos a respeito do subsistema suplementar pode impulsionar uma reflexão crítica a respeito da formação desses profissionais. Entender como essa formação em saúde caminha, seus desafios e limites, leva-nos a refletir sobre a formação que desejamos, que é a integralidade da atenção e o trabalho em equipe (CECCIM et al., 2008).

Nesse sentido, observou-se, neste estudo, que a grade curricular da maioria dos cursos não oferece conteúdo teórico aos alunos em relação ao subsistema de saúde suplementar. A revisão das ementas das disciplinas mostrou que o ensino do subsistema de saúde suplementar não está explícito nas grades ou não está sendo contemplado nos cursos da área da saúde da USP/Ribeirão Preto.
Em suma, abordar o tema subsistema de saúde suplementar parece implicar uma mudança de paradigma no processo de formação dos profissionais de saúde, inserindo outros conceitos e práticas com relação ao cuidado à saúde das pessoas (SILVA \& SENA, 2008).É, assim, importante aprofundar a reflexão sobre os meios e os modos como a formação profissional vem ocorrendo, ou seja, se os conteúdos curriculares e as metodologias de ensino utilizadas possibilitam ao aluno apreender tanto os procedimentos técnicos indispensáveis ao exercício profissional, quanto desenvolver visão crítica em relação ao processo de trabalho e ao mundo que o rodeia (FILHO, 2004).

No que diz respeito à enfermagem, Silva e Sena (2008) ressaltam a importância da formação do enfermeiro com vistas à transformação do cuidado prestado e trazem, na proposta da integralidade do cuidado, o olhar de alunos, docentes e profissionais de saúde. Buscando essa integralidade, as transformações curriculares ocorridas a partir de 2001 com as Diretrizes Curriculares Nacionais para os cursos de graduação em Enfermagem (DCENF) estabeleceram novas competências e habilidades que deverão ser desenvolvidas pelos estudantes durante seu processo de aprendizado (BRASIL, 2001). Essa implantação implica o desafio de formar profissionais com competência técnica e política, considerando-os sujeitos sociais que possuem conhecimento, raciocínio, percepção e sensibilidade, tendo ainda a instituição formadora o compromisso de capacitação dos alunos para intervirem nos mais diversos contextos de cuidado à saúde, sendo estes muitas vezes incertos e complexos (FERNANDES et al., 2005).

$\mathrm{O}$ egresso do curso de enfermagem precisa ser promotor da saúde integral do ser humano, tendo sempre como base o rigor científico. Além disso, sua atuação deve estar pautada em princípios éticos, e os campos de práticas e estágios, ricos em situações concretas (FILHO, 2004).

Fernandes et al. (2005) apontam que nessa modalidade de ensino-aprendizagem é preciso oportunizar ao aluno o desenvolvimento da 
capacidade de agir com eficácia frente às mais diversas situações, tendo como base conhecimentos adquiridos previamente, porém não se limitando a estes. De acordo com as autoras, é relevante focar a aquisição de competências, ou seja, a capacidade de utilizar os conhecimentos adquiridos e integrá-los ou mobilizá-los, visando a sua resolução; refere-se ao saber agir e transformar a prática, num processo de reflexão crítica do aprendizado e intervenção efetiva na prática do cuidado.

\section{Considerações Finais}

O ensino do subsistema de saúde suplementar representa, ainda, uma lacuna nos cursos de graduação da área de saúde do campus de Ribeirão Preto da Universidade de São Paulo. Portanto, entende-se que existe uma necessidade de se repensarem os currículos, com a introdução desse tema, tendo em vista a importância, cada vez maior, de formar profissionais bem preparados, com ampla visão do sistema de saúde brasileiro, considerando os seus subsistemas. Isso se torna mais relevante quando se pensa que o primeiro empregador de um estudante recém-formado na área da saúde é, geralmente, o empregador privado, com suas relações regidas pelo subsistema de saúde suplementar. Dessa forma, é fundamental que o aluno de um curso da saúde, futuro profissional nessa área, conheça as características do sistema para que possa operar corretamente o direito à saúde.

\section{Referências Bibliográficas}

ALBUQUERQUE, Veronica Santos; BATISTA Rodrigo Siqueira; TANJI, Suzelaine; MOÇO, Edneia Tayt-Sohn Martuchelli. "Discipline Curricula in the Health Area: An Essay on Knowledge and Power". Interface Comunic., Saúde, Educ., vol. 13, n. 31, 2009, pp. 261-72.

BARDIN, Laurence. Análise de Conteúdo. Lisboa: Edições 70, 1979.

BRASIL. Constituição 1988. Constituição da República Federativa do Brasil: promulgada em 5 de outubro de 1988. Organização do texto: Juarez de Oliveira. 4 ed. São Paulo: Saraiva, 1990.

BRASIL. "Lei n. 9.656, de 3 de junho de 1998. Dispõe sobre os planos privados de assistência à saúde". Diário Oficial da União, Brasilia, DF, 4 jun. 1998a. Seção 1, p. 1.
BRASIL. "Lei n. 9.961, de 28 de janeiro de 2000. Cria a Agência Nacional de Saúde Suplementar ANS e dá outras providências". Diário Oficial da União, Brasília, DF, 29 jan. 2000. Seção 1, p. 5.

BRASIL. Conselho Nacional de Educação: Câmara de Educação Superior. "Resolução CNE/CES n. 3, de 7 de novembro de 2001. Institui Diretrizes Curriculares Nacionais do Curso de Graduação em Enfermagem". Disponível em: $<$ http://portal.mec.gov.br/cne/arquivos/ pdf/CES03.pd†. Acessado em 15 set. 2017.

CARVALHO, Eurípedes Balsanufo \& CEGíliO, L. "A Regulamentação do Setor de Saúde Suplementar no Brasil: a Reconstrução de uma História de Disputas". Cadernos de Saúde Pública, vol. 23, n. 9, 2007, pp. 2167-2177.

GECGIM, Ricardo Burg; ARMANI, Teresa Borgert; OLIVEIRA, Dora Lúcia Leideins Correa de; BILIBIO, Luiz Fernando; MORAES, Maurício; SANTOS, Naiane Dartora. Imaginários da Formação em Saúde no Brasil e os Horizontes da Regulação em Saúde Suplementar. Ciência e Saúde Coletiva, vol. 13, n. 5, set./out. 2008. Disponível em: <http:// www.scielo.br/scielo.php?script=sci_arttext\&pi$\mathrm{d}=$ S1413-81232008000500021\&lang $=\mathrm{pt}>$. Acessado em: 15 set. 2017.

COUTO, Rita Maria de Souza. Movimento Interdisciplinar de Designers Brasileiros em Busca de Educação Avançada. Tese (Doutorado em Educação), Pontifícia Universidade Católica do Rio de Janeiro, 1997.

FERNANDES, Josicélia Dumêt; XAVIER, Iara de Moraes; CERIBELLI, Maria Isabel Pedreira de Freitas; BIANCO, Maria Helena Cappo; MAEDA, Dirce; RODRIGUES, Michele. Diretrizes Curriculares e Estratégias para Implantação de uma Nova Proposta Pedagógica. Rev. Esc. Enferm. USP, vol. 39, n. 4, 2005, pp. 443-449.

FILHO, Antenor Amâncio. "Dilemas e Desafios da Formação Profissional em Saúde". Interface - Comunic, Saúde, Educ, vol. 8, n. 15, 2004, pp. 375-80.

GIL, Antônio Carlos. Métodos e Técnicas de Pesquisa Social. 4. ed. São Paulo: Atlas, 1994.

MORIN, Edgar. A Cabeça Bem Feita: Repensar a Reforma, Reformar o Pensamento. 15 ed. Rio de Janeiro: Bertrand Brasil, 2008.

. Os Sete Saberes Necessários à Educasão do Futuro. 11 ed. São Paulo: Cortez; Brasília, DF: Unesco, 2006a, 118 p.

MOSÉ, Viviane. "Educação: a Briga no Rio Está Errada". O Globo, 30 out. 2008.

OLIVEIRA, Sílvio Luis de. Tratado de Metodologia Cientifica: Projetos de Pesquisa, TGI, TCC, Monografia, Dissertações e Teses. 2 ed. São Paulo: Pioneira, 1999.

OPAS, Organização Pan-Americana da Saúde. "Regulação e Modelos Assistenciais na Saúde 
Suplementar" [Lançamento do $14^{\circ}$ volume da Série Técnica Desenvolvimento de Sistemas e Serviços de Saúde], 2009. Disponível em: <http://www.paho.org/bra/ index.php?option $=$ com_content\&view $=$ article\&i$\mathrm{d}=518$ :regulacao-e-modelos-assistenciais-na-saude-suplementar\&Itemid=843>. Acessado em 15 set. 2017.

PIETROBON, Louise; PRADO, Martha Lenise do \& CAETANO, João Carlos. "Saúde Suplementar no Brasil: o Papel da Agência Nacional de Saúde Suplementar na Regulação do Setor". Physis, vol. 18, n. 4, 2008, pp. 767-783.

RESOLUÇÃO N. 3745, DE 19 DE OUTUBRO DE 1990. Regimento Geral da Universidade de São Paulo. Disponível em: <http://citrus.uspnet.usp.br/leginf/rg/ rg.html >. Acessado em 15 set. 2017.

RESOLUÇÃO CoG N. 4749, DE 17 DE ABRIL DE 2000. Disponível em: <http://www.leginf.usp. $\mathrm{br} /$ ?resolucao=resolucao-cog-no-4749-de-17-de-abrilde-2000>. Acessado em 15 set. 2017.

SALVATORI, Rachel Torres. O Papel da Agência Nacional de Saúde na Mediasão Ativa de Conflitos frente à Questão da Judicialização da Saúde: a Situação da Saúde Mental Suplementar no Brasil. Tese (Doutorado em Enfermagem), Escola de Enfermagem de Ribeirão Preto, Universidade de São Paulo, Ribeirão Preto, 2013.

SATO, Fabio Ricardo Loureiro. "A Teoria da Agência no Setor da Saúde: o Caso do Relacionamento da Agência Nacional de Saúde Suplementar com as Operadoras de Planos de Assistência Supletiva no Brasil". RAP, vol. 41, n. 1, 2007, pp. 49-62.

SILVA, Kênia Lara \& SENA, Roseni Rosângela. "Integralidade do Guidado na Saúde: Indicações a partir da Formação do Enfermeiro". Rev. Esc. Enferm USP, vol. 42, n. 1, 2008, pp. 48-56.

Publicado em 04/07/2018. 